\title{
Growth delay of human bladder cancer cells by Prostate Stem Cell Antigen downregulation is associated with activation of immune signaling pathways
}

\author{
Emanuele Marra', Paolo Uva ${ }^{1}$, Valentina Viti ${ }^{1}$, Valeria Simonelli ${ }^{3}$, Eugenia Dogliotti ${ }^{3}$, Emanuele De Rinaldis ${ }^{4}$,
} Armin Lahm', Nicola La Monica', Alfredo Nicosia², Gennaro Ciliberto', Fabio Palombo ${ }^{1 *}$

\begin{abstract}
Background: Prostate stem cell antigen (PSCA) is a glycosylphosphatidylinositol (GPI) anchored protein expressed not only in prostate but also in pancreas and bladder cancer as shown by immunohistochemistry and mRNA analysis. It has been targeted by monoclonal antibodies in preclinical animal models and more recently in a clinical trial in prostate cancer patients. The biological role played in tumor growth is presently unknown. In this report we have characterized the contribution of PSCA expression to tumor growth.

Methods: A bladder cell line was engineered to express a doxycycline (dox) regulated shRNA against PSCA. To shed light on the PSCA biological role in tumor growth, microarray analysis was carried out as a function of PSCA expression. Expression of gene set of interest was further analyzed by qPCR

Results: Down regulation of the PSCA expression was associated with reduced cell proliferation in vitro and in vivo. Mice bearing subcutaneous tumors showed a reduced tumor growth upon treatment with dox, which effectively induced shRNA against PSCA as revealed by GFP expression. Pathway analysis of deregulated genes suggests a statistical significant association between PSCA downregulation and activation of genes downstream of the IFN $/ \beta$ receptor.
\end{abstract}

Conclusions: These experiments established for the first time a correlation between the level of PSCA expression and tumor growth and suggest a role of PSCA in counteracting the natural immune response.

\section{Background}

PSCA has been discovered a decade ago and has been classified as a member of the Ly- 6 family of GPIanchored cell surface proteins [1]. It is expressed in most prostate cancer specimens, including high-grade prostatic intraepithelial neoplasia, primary androgendependent tumors, and hormone-refractory metastases. PSCA levels are positively correlated with Gleason grade, tumor stage, and biochemical recurrence. Its expression is also particularly elevated in bone metastasis. Finally, PSCA is strongly expressed in other malignancies, including bladder and pancreatic cancers [2-6].

\footnotetext{
* Correspondence: fabiopalombo@libero.it
'Istituto di Ricerche Biologia Molecolare P. Angeletti (IRBM), Via Pontina Km

* Correspondence: fabiopalombo@libero.it
${ }^{1}$ Istituto di Ricerche Biologia Molecolare P. Angeletti (IRBM), Via Pontina Km 30,600 00040 Pomezia (Rome) Italy
} 30,600 00040 Pomezia (Rome) Italy

Different immunotherapy approaches targeting PSCA have been tested in preclinical models including cancer vaccine, therapeutic monoclonal antibodies and antibody conjugated to toxic drugs [7-10]. More recently, a human monoclonal antibody targeting PSCA has been evaluated in a phase I clinical trial in prostate cancer patients (AACR 2006).

Little information is available regarding the biological role of PSCA. Proteins belonging to the Ly6 family are involved in cell signaling events associated with thymic lymphocyte differentiation, maturation and activation. CD59, a member of this protein family, was shown to play a role in the protection against complement mediated lysis. In addition, it was found to be expressed in tumor cells where it may play a role in evading anti 
cancer immune response [11]. Deletion of PSCA gene does not appear to interfere with normal development as PSCA knockout mice are viable. Additionally, crossing of the PSCA knockout mice with prostate tumor models driven by large $T$ antigen did not increase primary tumor formation $[12,13]$.

Here the biological role of human PSCA was evaluated using RNA interference and microarray analysis. To establish a pharmacologic control over gene expression a shRNA against PSCA was identified and expressed under the control of dox in a lentivirus system [14]. Microarray analysis was utilized to identify genes coregulated with PSCA in tumor xenografts.

\section{Methods}

\section{Cell culture and generation of lentivirus vectors}

The 293T and SW780 cell lines were cultured in Dulbecco's modified Eagle's medium supplemented with $10 \%$ fetal calf serum. The lentivirus system for conditional gene suppression with dox-inducible shRNAs utilized has been described previously[14]. Briefly, in the absence of dox, tTR-KRAB repressor binds to tet $O$ and suppresses H1-mediated shRNA transcription, thus allowing normal expression of the cellular target gene. In the presence of $10 \mu \mathrm{g} / \mathrm{ml}$ of dox, tTR-KRAB cannot bind to tet $O$ and hence shRNAs are produced, leading to downregulation of PSCA. The green fluorescent protein (GFP) cDNA contained in the shRNA vector provides a monitoring device, as it is switched on by dox treatment and GFPis expressed. All recombinant lentiviruses were produced by transient transfection in $293 \mathrm{~T}$ cells. Briefly, 293T cells were cotransfected with $20 \mu \mathrm{g}$ of pLUTHM-shPSCA3 plasmid, $15 \mu \mathrm{g}$ of pCMV$\Delta \mathrm{R} 8.91$, and $5 \mu \mathrm{g}$ of pMD2G-VSVG by calcium phosphate precipitation. After $16 \mathrm{~h}$ medium was changed, and recombinant lentivirus vectors were harvested $48 \mathrm{~h}$ later. FACS analysis was conducted as previously described [15].

\section{Viability assay}

Cell viability was monitored using the CellTiter-Blue Viability. The assay is based on the ability of living cells to convert a redox dye (resazurin) into a fluorescent end product (resorufin); $1 \times 10^{3}$ SW780-shPSCA and SW780shControl cells +/- Dox were plated in a 96 well plate in parallel with the parental cell line SW780. Cells were incubated at $37^{\circ} \mathrm{C}$ for $96 \mathrm{hrs}$, and fluorescence was subsequently monitored using a plate-reading fluorometer.

\section{Tumor models}

Six-week-old female CD-1 nude mice were purchased from Charles River Laboratories and maintained in accordance to Guidelines for the Care and Use of Laboratory Animals in IRBM's animal facility. This study, was submitted and approved by the IRBM ethical committee . Mice were injected subcutaneously (sc) in the right flank with $2 \times 10^{6} \mathrm{SW780}$-shPSCA cells resuspended into $100 \mu \mathrm{L}$ phosphate-buffered saline (PBS) and Matrigel (1:1). Mice received $5 \%$ sucrose only or $5 \%$ sucrose plus $0.2 \mathrm{mg} / \mathrm{ml}$ of dox for control and knockdown cohorts, respectively. All water bottles were changed 3 times per week. Tumors were measured with calipers and mice weighed twice per week. At the end of the dosing study, or as indicated, appropriate tumor samples were taken.

\section{Microarray experiments}

Total RNA from cell in culture was isolated with RNAzolB and then dissolved in RNase-free water. $25 \mu \mathrm{g}$ of total RNA was treated with DNase using the Qiagen RNase-free DNase kit and RNeasy spin columns. Then RNA was dissolved in RNase-free water to a final concentration of $0.2 \mu \mathrm{g} / \mu \mathrm{l}$. cRNA was generated using T7 RNA polymerase on $5 \mu \mathrm{g}$ of total RNA and labeled with Cy5 or Cy3 (Cy Dye, Amersham Pharmacia Biotech). From each sample, $5 \mu \mathrm{g}$ of labeled-RNA were co-hybridized with $5 \mu \mathrm{g}$ of a reference RNA (pool of two untreated SW780 cell lines). Labeled cRNAs were fragmented to an average size of 50-100 nucleotides by heating the samples to $60^{\circ} \mathrm{C}$ with $10 \mathrm{mM}$ of zinc chloride and then adding an hybridization buffer containing 1 $\mathrm{M} \mathrm{NaCl}, 0.5 \%$ sodium sarcosine, $50 \mathrm{mM}$ MES, pH6.5, and formamide to a final concentration of $30 \%$. The final volume was $3 \mathrm{ml}$ at $40^{\circ} \mathrm{C}$.

Samples were hybridized on a customized Agilent $44 \mathrm{k}$ array containing $\sim 40,000$ unique probes mapping to $\sim 21,000$ human genes. Each sample was hybridized in duplicate with fluor reversal to systematically correct for dye bias. After hybridization, slides were washed and scanned using a confocal laser scanner (Agilent Technologies). The raw intensities obtained after scanning were quantified, background-corrected and lowness normalized. A weighted average ratio was computed for dye-swapped pairs of hybridizations. Tumors were collected in RNA later and processed for RNA extraction as described previously [16]. Samples were hybridized on a customized Affymetrix array containing $\sim 38,000$ probes mapping to $\sim 21,000$ human genes [17].

\section{Hierarchical clustering}

The microarray dataset was filtered before clustering in order to select the 2,000 most variable probes. probes with an absent call in more than 12 samples out of 14 were removed the 2,000 probes with the higher interquartile range were retained for subsequent analysis. Probes were hierarchically clustered using an average linkage algorithm based on Pearson correlation coefficients. 


\section{Identification of deregulated probes}

Differences in average probe expression between the dox + (PSCA silencing) and dox-samples were computed by 1-way ANOVA. Probes differentially expressed between the two classes were identified based on ANOVA p-value $<0.001$.

\section{Gene set enrichment analysis}

Groups of genes identified by 1-way ANOVA were compared to a collection of annotated gene sets to identify the functional classes that were significantly over-represented. The enrichment $\mathrm{p}$-values were computed according to the Fisher's exact test. The gene sets were obtained from public (Gene Ontology [18], KEGG [19], Interpro [20], Panther [21], oPOSSUM [22] and commercial sources (GeneGo (GeneGo Inc., St Joseph, MI, USA), Ingenuity (Ingenuity Systems Inc, Mountain View, CA, USA), TRANSFAC [23].

\section{RT-PCR}

Microarray findings were confirmed by real-time reverse-transcription PCR (RT-PCR) using the average value of mRNA from dox untreated SW780-shPSCA samples as calibrator. Analysis of mRNA expression of selected IFNa genes was performed using Applied Biosystems expression kit following manufacturers' instructions. Expression values were computed using the comparative CT method ( $\Delta \Delta \mathrm{CT}$ ) with GAPDH gene expression value serving as normaliser.

\section{Results}

Establishment of cell clones with inducible shRNA against PSCA

Published studies on PSCA have reported limited expression in cell lines engineered, for the most part, to express this protein ectopically $[7,10]$. Searching in a microarray cell atlas comprising 20 cell lines of different origins including prostate, pancreas and bladder revealed a low frequency of PSCA expression. The few cell lines with a detectable level of PSCA mRNA scored negative when expression was analyzed at protein level by FACS analysis (data not shown), suggesting that display of PSCA on the cell surface is uncommon. We therefore utilized a bladder cell line, SW780, which was shown to express PSCA on the cell surface [8]. The cell line was engineered to express different shRNA sequences against PSCA (Table 1) or a control shRNA against luciferase. To achieve a pharmacological controlled expression a dox inducible promoter was utilized in the context of a lentivirus system [14]. One out of six shRNA against PSCA showed a clear down regulation of target gene expression and was therefore utilized to generate SW780-shPSCA, which expresses also the tet regulator. Dox treatment resulted in $75 \%$ reduction of PSCA
Table 1 shRNA sequences against PSCA

\begin{tabular}{clll}
\hline shrNA & \multicolumn{1}{c}{ Sequence } & Coding Region & N \\
\hline 1 & CATCCTAACGCAAGTCTGATT & No & $497-515$ \\
\hline 2 & GCAAGTCTGACCATGTATGTT & No & $506-524$ \\
\hline 3 & GGCAGATCAGCTCTAGTGATT & No & $585-603$ \\
\hline 4 & CAAGTCTGACCATGTATGTTT & No & $507-525$ \\
\hline 5 & GCAAGAAGAACATCACGTGTT & Yes & $256-274$ \\
\hline 6 & GTGACACCGACTTGTGCAATT & Yes & $277-295$ \\
\hline
\end{tabular}

expression as measured by FACS analysis. Since PSCA is a membrane protein a further step was undertaken to improve regulation of gene expression by sorting out cells with low PSCA expression in the presence of dox. This process led to the isolation of cells with a larger interval of regulation mediated by dox treatment. To control that the expression of shRNA was induced upon dox treatment we took advantage of GFP gene, which is cloned upstream of the shRNA and it was previously utilized as a marker of shRNA expression [14]. FACS analysis showed a quantitative GFP expression only in dox treated SW780-shPSCA cells and a strong reduction of PSCA expression (Figure 1A). These data suggest that expression of shRNA specific for PSCA can be induced and that it leads to almost $90 \%$ downregulation of the target gene.

Biological effects of PSCA down-regulation in SW780 cells To characterize the impact of PSCA expression on cell growth, a viability assay was carried out. A statistical significant reduction in cell viability was observed in dox treated cells whereas no difference was observed in control transfected cells (Figure 1B).

To verify the impact of PSCA expression on SW780shPSCA tumor growth dox treatment was either started in vitro one week in advance or at the time of cell implantation. A statistical significant reduction in s.c. tumor growth $(\mathrm{p}<0.05)$ was observed in the cohort of animals treated with dox with respect to controls including also SW780-shCont treated with dox (Figure 2A). Interestingly, tumors generated with cells pretreated in vitro with dox were smaller than tumors treated with dox only in vivo, although this difference was not statistically significant. In contrast control shRNA did not show any statistical reduction of tumor growth. To confirm that shRNA against PSCA was induced in vivo tumors were sacrificed at day 28 and expression of shRNA was evaluated by looking at GFP expression. Only dox-treated mice showed GFP expression in SW780-shPSCA tumors (Figure 2B). In line with the shRNA expression, PSCA was down-regulated as shown by IHC. All together these experiments indicate that PSCA plays a critical role in the SW780-shPSCA 


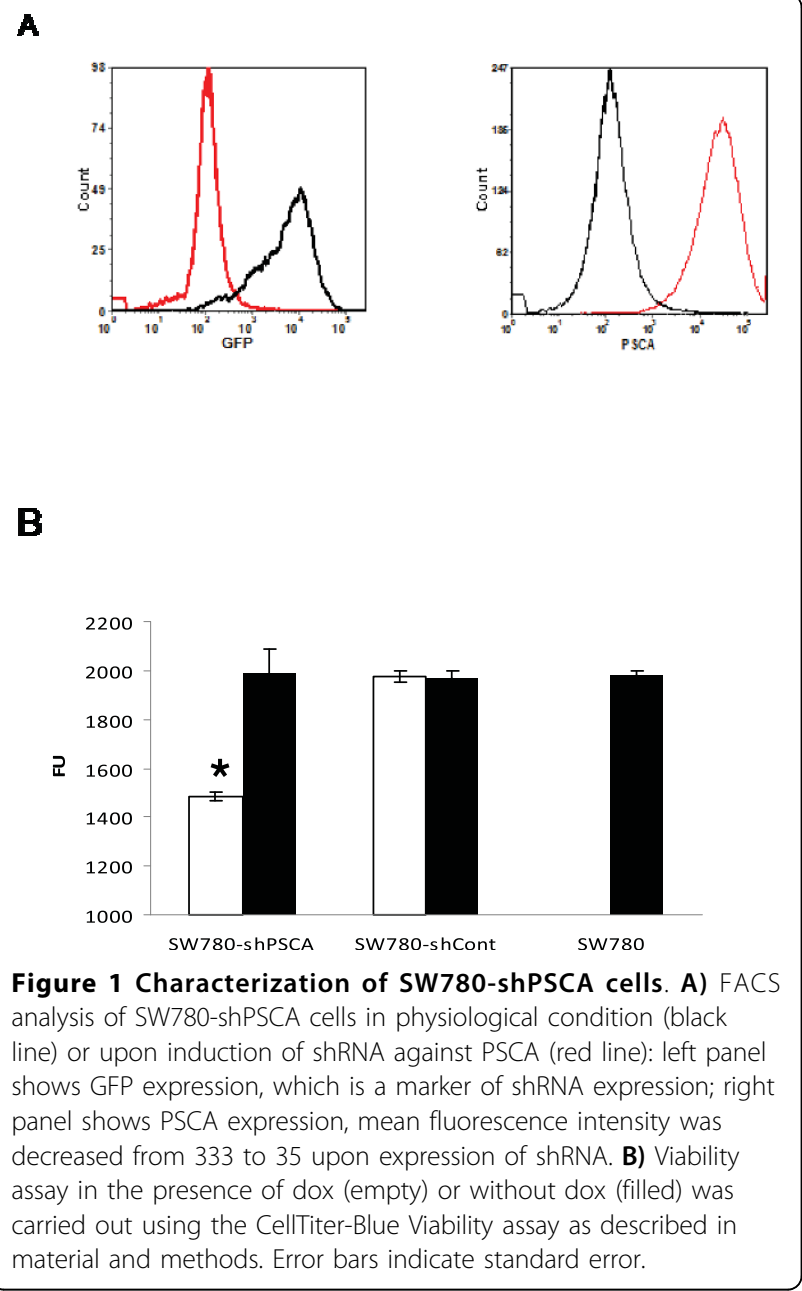

tumor model leading to more than $50 \%$ reduction of tumor mass with respect to dox untreated tumors.

\section{Transcriptional data analysis}

To gain some hints on the biological role played by PSCA, expression profile was carried out in tumors. Unsupervised hierarchical clustering of genome-wide expression data showed that samples clustered according to dox treatment (Figure 3A), thus demonstrating a clear effect of the PSCA expression levels on transcriptional programs. To identify the genes differentially expressed in the two experimental conditions, a 1-way ANOVA analysis was carried out. Out of the 37,586 probes represented on the array, 284 and 353 were found to be respectively up- and down-regulated in dox+ samples, as compared to dox-samples (ANOVA p-value $<0.001)$. Similar microarray experiments were also conducted in vitro showing a similar separation between the two groups, according to dox treatment. Comparison between the in vitro and in vivo data sets revealed a statistical significant overlap for the overexpressed genes (Table 2), although it may represent an underestimation due to the limited size of in vitro observations.

The genes identified as differentially expressed were analyzed by gene set enrichment analysis, using the same method also used elsewhere [24]. In this way we could monitor the pathways showing a global transcriptional perturbation upon silencing of PSCA (Figure 3B). We defined as up- or down-regulated the pathways statistically enriched in the list of genes up- or downregulated, respectively, in the presence or absence of treatment. A statistically significant enrichment was observed between the list of genes up-regulated upon PSCA silencing (dox +$)$. The IFN $\alpha / \beta$ signaling pathway resulted to be up-regulated upon PSCA silencing in the in-vivo experiments but its up-regulation was not evident in the in vitro datase. Out of the 27 genes listed in the Ingenuity database for interferon signaling 12 were up-regulated in vivo and 1 in vitro, STAT2. Similar results were obtained using the GeneGo database to run the analyses ( 8 out of 28 genes). In addition, the "antigen presentation" pathway resulted as perturbed with the highest statistical significance. Other immune related pathways were also affected although at lower statistical significance. In agreement with the reduced cell viability in vitro (Figure $1 \mathrm{~B}$ ) and reduced tumor growth (Figure 2A) the down-regulated pathways were "growth" and "negative regulation of apoptosis".

To measure by a more quantitative method the amplitude of gene regulation, selected genes were analyzed by qRT-PCR. As shown in Figure 4A, a subset of genes belonging to IFN $\alpha / \beta$ pathway such as IFIT1, IFI27 and IFI44L showed a statistical significant up-regulation $(\mathrm{p}<$ 0.001) in tumors where PSCA was down-regulated by dox treatment. To exclude that expression of shRNA per se could trigger expression of IFN pathway genes, tumors expressing control shRNA were analyzed. No statistical differences were observed with or without control shRNA expression. Interestingly, qPCR analysis of SW780-shPSCA in cell culture (Figure 4B) confirmed the lack of induction of the IFNa genes regardless of PSCA expression, suggesting a distinct role for PSCA in the context of tumor growth.

\section{Discussion}

In this study, we have identified a correlation between PSCA expression and tumor growth in vivo. High level of PSCA mRNA and protein expression has been observed in most primary prostate and pancreatic human tumors and in particular in aggressive metastatic forms. In bladder cancer, higher levels of PSCA expression correlated with increasing tumor grade [25] and more recently genetic variants in this gene were associated with cancer occurrence, although the biological 
$\mathbf{A}$

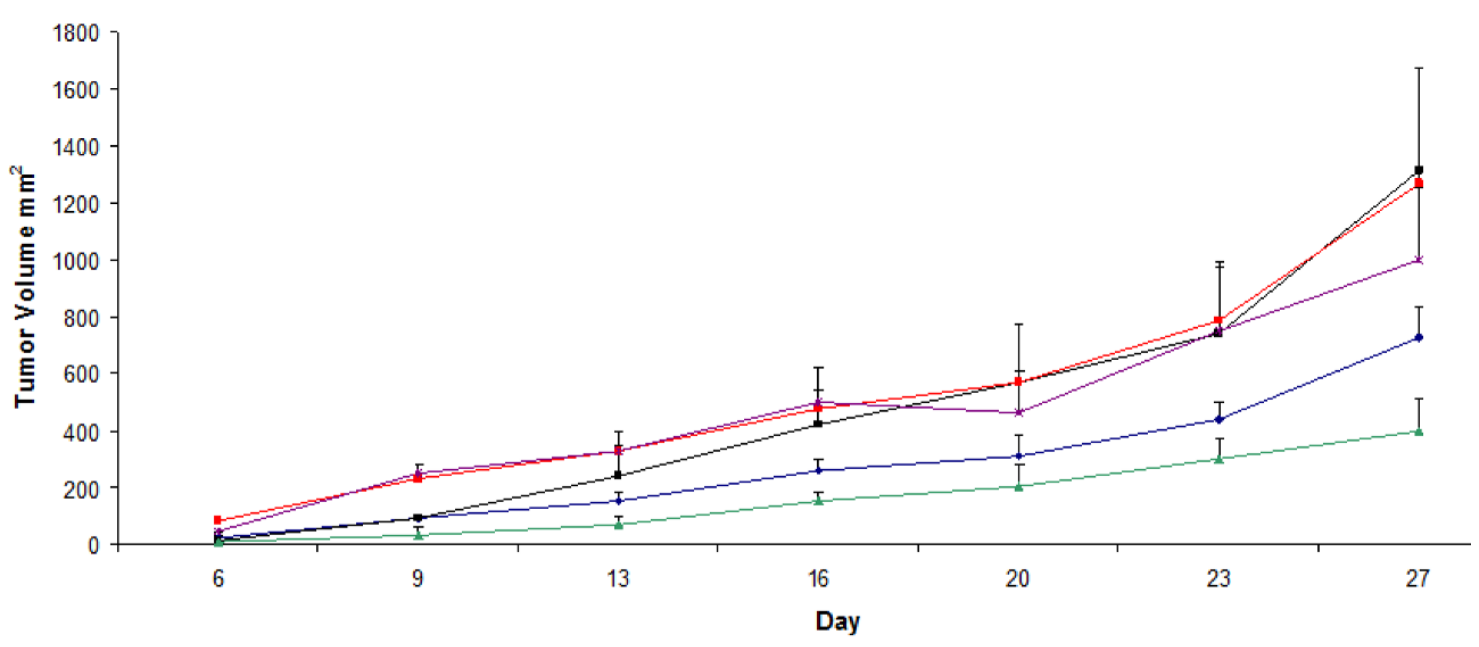

B

PSCA

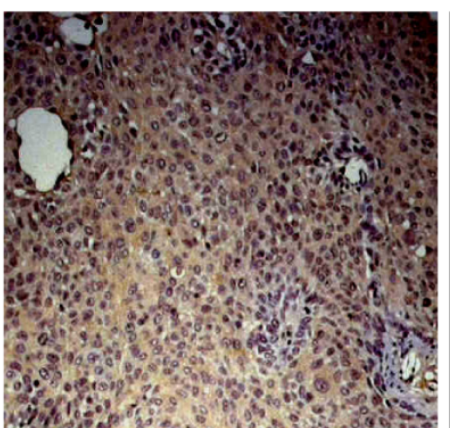

-Dox

+ Dox

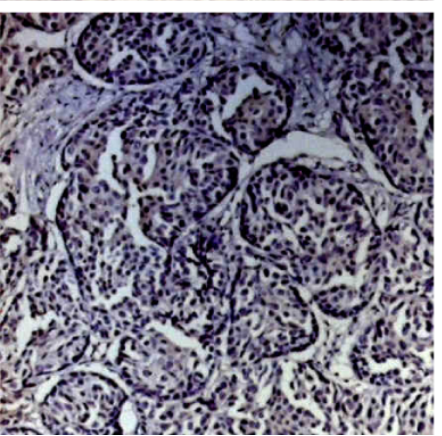

GFP
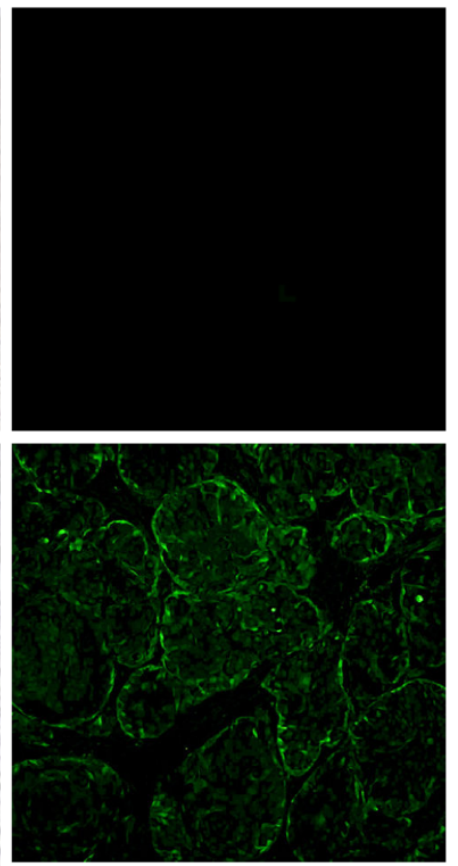

Figure 2 SCA silencing inhibits SW780-shPSCA tumor growth. A) Balb/C nude mice were injected s.c. with SW780-shPSCA cells or SW780shCont cells. To further lower PSCA expression a group of mice was injected with SW780-shPSCA cells pretreated with dox in vitro for 1 week (green line). Groups of 6-10 mice were injected with cells and treated as follow: SW780-shPSCA bearing mice not treated with dox (red) or treated with dox (green and blue); SW780-shCont. Treated with dox (magenta) or not treated (black). B) At the end of the experiment SW780shPSCA tumors were collected and subjected to IHC analysis for PSCA expression (left panel) or analyzed for GFP expression (right panel). 


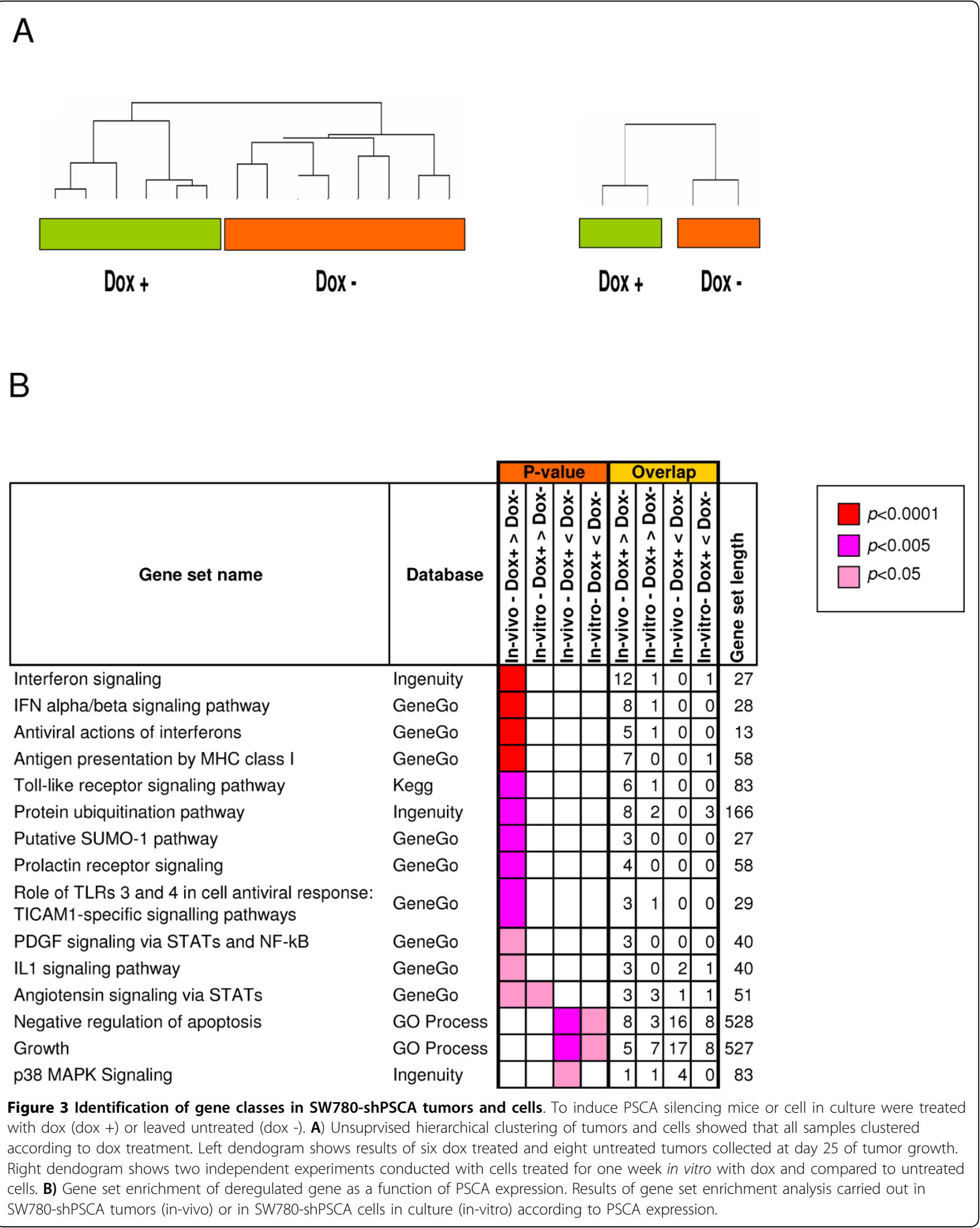


Table 2 Genes differentially expressed upon reduction of PSCA expression and overlap between in vitro and in vivo dataset.

\begin{tabular}{ccccc}
\hline Genes & in vitro & in vivo & overlap & p-value \\
\hline up regulated & 123 & 208 & 8 & 0.0001 \\
\hline down regulated & 119 & 234 & 3 & 0.19 \\
\hline
\end{tabular}

implications of this observation remain to be elucidated [26].

The observed tumor specific expression of PSCA has prompted the development of therapeutic antibodies specific for this membrane protein. Indeed, growth inhibition has been observed upon antibody treatment of cells transfected with vectors expressing PSCA or of naturally expressing cell lines such as SW780. The published studies are in agreement with the data reported here indicating that reduced PSCA expression is associated with lower viability [7]. Thus, based on these published data, a better understanding of the biologic role played by PSCA in tumor growth is warranted.

To shed light on PSCA role in tumor biology we compared PSCA expression in different cell lines and found expression on cell surface in few of them or in explanted primary tumors. Thus, the bladder cell line SW780 was engineered to obtain a pharmacological controlled expression of PSCA and to study the impact of reduced PSCA gene expression on cell viability and tumor growth. Here, we report a direct correlation between PSCA expression and tumor growth. Of note is the observation that pretreatment with dox in vitro further reduced tumor growth. This latter result may be partially explained by the slow decay of PSCA displayed on the cell membrane as indicated by FACS analysis.

A role for PSCA in tumor proliferation is further supported by the biological pathway analysis of gene expression profiling where a statistical significant association with "negative regulation of apoptosis" and "growth" was observed. Many of the pathways activated upon PSCA downregulation control key immune functions. Activation of IFN $\alpha / \beta$ signal transduction pathway was observed only in SW780-shPSCA tumors (Figure 2B) and it was not evident in tumors obtained upon injection of control cells. In contrast, similar studies conducted with these cell lines in vitro did not show induction of the pathway suggesting that in the tumor context additional factors such as those related to immune modulation may contribute to the observed microarray signature. A quantitative analysis of gene expression by qPCR confirmed overexpression of IFN $\alpha / \beta$ genes (Figure 4 ). The activation of IFN $\alpha / \beta$ pathway only in vivo further supports the idea that triggering of this pathway is not determined by an intracellular mechanism related to double strand RNA, as it has been previously reported. On the contrary, the data suggest that IFN $\alpha / \beta$ activation is related to environmental signals. The activation of IFN $\alpha / \beta$ pathway is consistent with a reduced tumor growth as previously shown with recombinant IFN $[27,28]$.

Nonetheless, further experiments are required to better characterize the link between PSCA downregulation and activation of immune pathways such as IFN $\alpha / \beta$. In this direction it is worth mentioning a potential physical

A

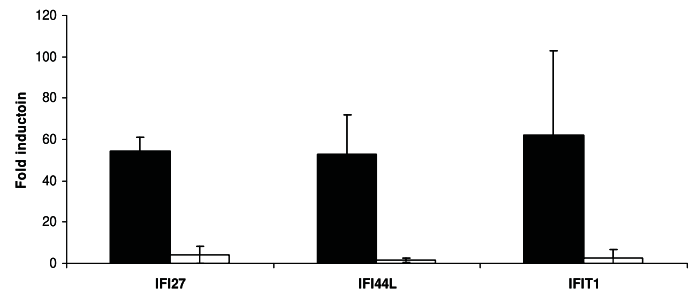

B

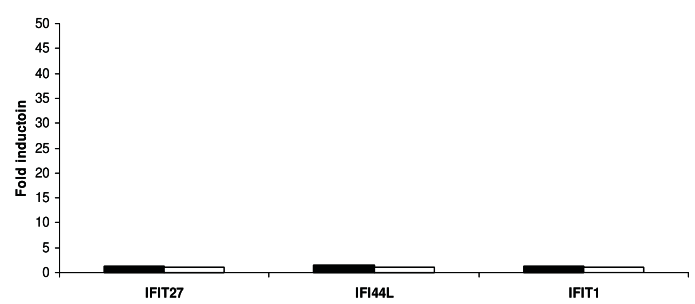

Figure 4 Expression levels of selected IFN $\alpha / \beta$ pathway genes as a function of PSCA expression. Expression levels for IFI27, IFI44L and IFIT1 genes were measured in individual SW780-shPSCA tumors $(n=6-8)(\mathbf{A})$ or in SW780-shPSCA in cell culture conditions (B) with or without dox treatment. Gene expression was measured by qPCR using the $\triangle \triangle \mathrm{ct}$ method. The average gene values in dox untreated mice served as reference and GAPDH gene as normalizer. In black average values in SW780-shPSCA tumors and in white SW780-shCont. 
interaction between PSCA and IFN $\alpha / \beta$ receptor. PSCA is a GPI-anchored protein located in lipid raft. IFN $\alpha / \beta$ receptor can be brought into this subcellular compartment upon interaction with its ligands [29]. Thus, PSCA may counteract intracellular signaling exerted by the IFN $\alpha / \beta$ receptor playing a role as a defense protein. This biological role is reminiscent of the interfering role played by PSCA homolog, CD59, in the complement system [30]. Activation of the complement system is tightly regulated and among the various regulators CD59 has been identified as the single membrane regulator of the terminal membrane attack complex. Moreover CD59 has been characterized as a negative regulator of the $\mathrm{T}$-cell response in mice. Although we limited our qPCR confirmation only to IFN $\alpha / \beta$ pathway, a statistical significant up regulation was observed also for other immune pathways such as of MHC-1 pathway. Given the pleiotropic effects of IFN $\alpha / \beta$ pathway it is likely that some pathways, including $\mathrm{MHC}-1$ presentation, may be affected downstream. A more in depth analysis would be required to answer this question. Finally we cannot exclude that the correlations observed only in tumors and not in cell culture are driven by undefined factors that are not captured in the expression profile and deserve further investigation.

\section{Conclusions}

The current findings suggest a role of PSCA in counteracting the natural immune response against cancer. This information could help in design more effective experiments in preclinical models not only with monoclonal antibodies but also with approaches based on cancer vaccine [31-35]. It would be of interest to correlate antitumor efficacy of PSCA-targeting therapies as a function of the natural immune response.

\section{Acknowledgements}

The authors thank Aya Jakobovits and Jean Gudas for anti PSCA antibody, 4.117. The authors also thank Brendan Leeson and Ernest Coffey from Gene Expression Laboratories - Rosetta and LAR personnel for technical support. This work was partially supported by Progetto Integrato Oncologia, Ministero della Salute e Associazione Italiana per la Ricerca sul Cancro (AIRC).

\section{Author details}

'Istituto di Ricerche Biologia Molecolare P. Angeletti (IRBM), Via Pontina Km 30,600 00040 Pomezia (Rome) Italy. ${ }^{2}$ Okairos, Via dei Castelli Romani 22 00040 Pomezia (Rome) Italy. ${ }^{3}$ Istituto Superiore di Sanità (ISS), Viale Regina Elena 299, 00161 (Rome) Italy. ${ }^{4}$ Breakthrough Breast Cancer Research Unit, King's College London, UK

\section{Authors' contributions}

EM generated cell lines expressing shRNA, analyzed PSCA expression by FACS analysis and performed experiments in vivo. PU performed microarray analysis and pathway analysis- W generated cell lines expressing shRNA and performed IHC on tumor samples. VS performed qPCR analysis. ED contributed to the discussion of the data. EDR contributed in the discussion of the bioinformatics data and helped in drafting bioinformatics result section. AL performed the initial microarray analysis and contributed to data analysis. NLM, AN and GC contributed to the drafting and general discussion of the paper. FP conceived the study, analyzed the data and wrote the manuscript. All authors read and approved the final manuscript.

\section{Competing interests}

The authors declare that they have no competing interests.

Received: 25 November 2009 Accepted: 7 April 2010

Published: 7 April 2010

\section{References}

1. Reiter RE, Gu Z, Watabe T, Thomas G, Szigeti K, Davis E, Wahl M, Nisitani S, Yamashiro J, Le Beau MM, et al: Prostate stem cell antigen: a cell surface marker overexpressed in prostate cancer. Proc Natl Acad Sci USA 1998 95:1735-1740.

2. Rodriguez JA, Li M, Yao Q, Chen C, Fisher WE: Gene overexpression in pancreatic adenocarcinoma: diagnostic and therapeutic implications. World J Surg 2005, 29:297-305.

3. Elsamman E, Fukumori T, Kasai T, Nakatsuji H, Nishitani MA, Toida K, Ali N, Kanayama $\mathrm{HO}$ : Prostate stem cell antigen predicts tumour recurrence in superficial transitional cell carcinoma of the urinary bladder. BJU Int 2006, 97:1202-1207.

4. Grubbs EG, Abdel-Wahab Z, Tyler DS, Pruitt SK: Utilizing quantitative polymerase chain reaction to evaluate prostate stem cell antigen as a tumor marker in pancreatic cancer. Ann Surg Oncol 2006, 13:1645-1654.

5. Olafsen T, Gu Z, Sherman MA, Leyton JV, Witkosky ME, Shively JE, Raubitschek AA, Morrison SL, Wu AM, Reiter RE: Targeting, imaging, and therapy using a humanized antiprostate stem cell antigen (PSCA) antibody. J Immunother 2007, 30:396-405.

6. Tanaka M, Komatsu N, Terakawa N, Yanagimoto Y, Oka M, Sasada T, Mine T, Gouhara S, Shichijo S, Okuda S, Itoh K: Increased levels of IgG antibodies against peptides of the prostate stem cell antigen in the plasma of pancreatic cancer patients. Oncol Rep 2007, 18:161-166.

7. Gu Z, Yamashiro J, Kono E, Reiter RE: Anti-prostate stem cell antigen monoclonal antibody $1 \mathrm{G} 8$ induces cell death in vitro and inhibits tumor growth in vivo via a Fc-independent mechanism. Cancer Res 2005, 65:9495-9500.

8. Ross S, Spencer SD, Holcomb I, Tan C, Hongo J, Devaux B, Rangell L, Keller GA, Schow P, Steeves RM, et al: Prostate stem cell antigen as therapy target: tissue expression and in vivo efficacy of an immunoconjugate. Cancer Res 2002, 62:2546-2553.

9. Saffran DC, Raitano AB, Hubert RS, Witte ON, Reiter RE, Jakobovits A: AntiPSCA mAbs inhibit tumor growth and metastasis formation and prolong the survival of mice bearing human prostate cancer xenografts. Proc Natl Acad Sci USA 2001, 98:2658-2663.

10. Wente MN, Jain A, Kono E, Berberat PO, Giese T, Reber HA, Friess $H$, Buchler MW, Reiter RE, Hines OJ: Prostate stem cell antigen is a putative target for immunotherapy in pancreatic cancer. Pancreas 2005, 31:119-125.

11. Gorter A, Meri S: Immune evasion of tumor cells using membrane-bound complement regulatory proteins. Immunol Today 1999, 20:576-582.

12. Jalkut MW, Reiter RE: Role of prostate stem cell antigen in prostate cancer research. Curr Opin Urol 2002, 12:401-406.

13. Moore ML, Teitell MA, Kim Y, Watabe T, Reiter RE, Witte ON, Dubey P: Deletion of PSCA increases metastasis of TRAMP-induced prostate tumors without altering primary tumor formation. Prostate 2008 , 68:139-151.

14. Wiznerowicz M, Trono D: Conditional suppression of cellular genes: lentivirus vector-mediated drug-inducible RNA interference. J Virol 2003, 77:8957-8961.

15. Elia L, Mennuni C, Storto M, Podda S, Calvaruso F, Salucci V, Aurisicchio L, Scarito A, Ciliberto G, La Monica N, Palombo F: Genetic vaccines against Ep-CAM break tolerance to self in a limited subset of subjects: initial identification of predictive biomarkers. Eur J Immunol 2006, 36:1337-1349.

16. D'Errico M, Rinaldis ED, Blasi MF, Viti V, Falchetti M, Calcagnile A, Sera F, Saieva C, Ottini L, Palli D, et al: Genome-wide expression profile of sporadic gastric cancers with microsatellite instability. Eur J Cancer 2008, 45(3):461-9.

17. Irizarry RA, Bolstad BM, Collin F, Cope LM, Hobbs B, Speed TP: Summaries of Affymetrix GeneChip probe level data. Nucleic Acids Res 2003, 31:e15.

18. Ashburner M, Ball CA, Blake JA, Botstein D, Butler H, Cherry JM, Davis AP, Dolinski K, Dwight SS, Eppig JT, et al: Gene ontology: tool for the 
unification of biology. The Gene Ontology Consortium. Nat Genet 2000, 25:25-29.

19. Kanehisa M, Goto S: KEGG: kyoto encyclopedia of genes and genomes. Nucleic Acids Res 2000, 28:27-30.

20. Mulder NJ, Apweiler R, Attwood TK, Bairoch A, Bateman A, Binns D, Bork P, Buillard V, Cerutti L, Copley R, et al: New developments in the InterPro database. Nucleic Acids Res 2007, 35:D224-228.

21. Thomas PD, Campbell MJ, Kejariwal A, Mi H, Karlak B, Daverman R, Diemer K, Muruganujan A, Narechania A: PANTHER: a library of protein families and subfamilies indexed by function. Genome Res 2003, 13:2129-2141.

22. Ho Sui SJ, Mortimer JR, Arenillas DJ, Brumm J, Walsh CJ, Kennedy BP, Wasserman WW: oPOSSUM: identification of over-represented transcription factor binding sites in co-expressed genes. Nucleic Acids Res 2005, 33:3154-3164.

23. Matys V, Fricke E, Geffers R, Gossling E, Haubrock M, Hehl R, Hornischer K, Karas D, Kel AE, Kel-Margoulis OV, et al: TRANSFAC: transcriptional regulation, from patterns to profiles. Nucleic Acids Res 2003, 31:374-378.

24. Uva P, Aurisicchio L, Watters J, Loboda A, Kulkarni A, Castle J, Palombo F, Viti V, Mesiti G, Zappulli V, et al: Comparative expression pathway analysis of human and canine mammary tumors. BMC Genomics 2009, 10:135.

25. Amara N, Palapattu GS, Schrage M, Gu Z, Thomas GV, Dorey F, Said J, Reiter RE: Prostate stem cell antigen is overexpressed in human transitional cell carcinoma. Cancer Res 2001, 61:4660-4665.

26. Wu X, Ye Y, Kiemeney LA, Sulem P, Rafnar T, Matullo G, Seminara D, Yoshida T, Saeki N, Andrew AS, et al: Genetic variation in the prostate stem cell antigen gene PSCA confers susceptibility to urinary bladder cancer. Nat Genet 2009, 41:991-995.

27. Rosebeck S, Leaman DW: Mitochondrial localization and pro-apoptotic effects of the interferon-inducible protein ISG12a. Apoptosis 2008, 13:562-572.

28. Santodonato L, Ferrantini M, Palombo F, Aurisicchio L, Delmastro P, La Monica N, Di Marco S, Ciliberto G, Du MX, Taylor MW, Belardelli F: Antitumor activity of recombinant adenoviral vectors expressing murine IFN-alpha in mice injected with metastatic IFN-resistant tumor cells. Cancer Gene Ther 2001, 8:63-72.

29. Claudinon J, Monier MN, Lamaze C: Interfering with interferon receptor sorting and trafficking: impact on signaling. Biochimie 2007, 89:735-743.

30. Kimberley FC, Sivasankar B, Paul Morgan B: Alternative roles for CD59. Mol Immunol 2007, 44:73-81.

31. Dannull J, Diener PA, Prikler L, Furstenberger G, Cerny T, Schmid U, Ackermann DK, Groettrup M: Prostate stem cell antigen is a promising candidate for immunotherapy of advanced prostate cancer. Cancer Res 2000, 60:5522-5528.

32. Thomas-Kaskel AK, Zeiser R, Jochim R, Robbel C, Schultze-Seemann W, Waller CF, Veelken $\mathrm{H}$ : Vaccination of advanced prostate cancer patients with PSCA and PSA peptide-loaded dendritic cells induces DTH responses that correlate with superior overall survival. Int J Cancer 2006, 119:2428-2434.

33. Morgenroth A, Cartellieri M, Schmitz M, Gunes S, Weigle B, Bachmann M, Abken H, Rieber EP, Temme A: Targeting of tumor cells expressing the prostate stem cell antigen (PSCA) using genetically engineered T-cells. Prostate 2007, 67:1121-1131.

34. Zhang X, Yu C, Zhao J, Fu L, Yi S, Liu S, Yu T, Chen W: Vaccination with a DNA vaccine based on human PSCA and HSP70 adjuvant enhances the antigen-specific CD8+ T-cell response and inhibits the PSCA+ tumors growth in mice. J Gene Med 2007, 9:715-726.

35. Garcia-Hernandez Mde L, Gray A, Hubby B, Klinger OJ, Kast WM: Prostate stem cell antigen vaccination induces a long-term protective immune response against prostate cancer in the absence of autoimmunity. Cancer Res 2008, 68:861-869.

\section{Pre-publication history}

The pre-publication history for this paper can be accessed here: http://www. biomedcentral.com/1471-2407/10/129/prepub

doi:10.1186/1471-2407-10-129

Cite this article as: Marra et al:: Growth delay of human bladder cancer cells by Prostate Stem Cell Antigen downregulation is associated with activation of immune signaling pathways. BMC Cancer 2010 10:129.

\section{Submit your next manuscript to BioMed Central and take full advantage of:}

- Convenient online submission

- Thorough peer review

- No space constraints or color figure charges

- Immediate publication on acceptance

- Inclusion in PubMed, CAS, Scopus and Google Scholar

- Research which is freely available for redistribution 